\title{
Stability results for sets of uniqueness in binary tomography
}

\author{
Paolo Dulio ${ }^{1, a}$ and Silvia M.C. Pagani ${ }^{1, b}$ \\ ${ }^{1}$ Dipartimento di Matematica "F. Brioschi", Politecnico di Milano, Piazza Leonardo da Vinci 32, I-20133 Milano
}

\begin{abstract}
The recovery of an unknown density function from the knowledge of its projections is the aim of tomography. In many cases, considering the problem from a discrete perspective is more convenient than employing a continuous approach: discrete tomography, and in particular binary tomography, is therefore exploited. One of the main goals of tomography is guaranteeing that the produced output coincides with the scanned object, namely, one wants to achieve uniqueness of reconstruction, even when only a few directions, from which projections are taken, are employed. Relying on a theoretical result stating that special sets of just four lattice directions are enough to uniquely reconstruct a binary grid, we prove that such sets are stable, in the sense that a small discrete perturbation of the components of the directions returns sets which again ensure uniqueness of reconstruction. Examples are provided.
\end{abstract}

\section{Introduction}

Tomography takes its practical foundation from the following request: reconstruct the interior of an object which is not directly accessible (for instance, a human brain) without opening and/or damaging it. Tomography is therefore a branch of the inverse problems, and was born thanks to the theoretical work done by Johann Radon [1], who gave a formula to reconstruct a function from its line integrals. This continuous approach is known as computed tomography. In order to achieve the reconstruction, X-rays are sent from a source to a detector, and what we measure is their attenuation coefficient, meaning that the ray encountered some kind of obstacle in its trajectory.

The continuous framework in which Radon inversion formula takes place, however, reveals not to be the best way of modeling reality. The most important drawback in the continuous approach is the necessity of considering projections from all angles in $[0,2 \pi)$, which is of course not achievable in the real world. This reflects into performing some kind of discretization, leading to discrete tomography, where integrals are replaced by finite sums, and different models can be employed according to the different ways of representing pixels and X-rays. As a special case of discrete tomography we have binary tomography, where one is interested in detecting the presence or the absence of the object itself. This paper deals with binary objects.

A fundamental issue of tomography is uniqueness of reconstruction, namely, one wants to ensure that the reconstructed object is exactly the original one. Generally speaking, the reconstruction problem is ill-posed. Ambiguities descend from the fact that the Radon transform is

\footnotetext{
a e-mail: paolo.dulio@polimi.it

be-mail: silviamaria.pagani@polimi.it
}

injective only if projections are available for all angles in $[0,2 \pi)$. If some angles are missing, then the kernel of the Radon transform is not trivial. In the discrete case, this reflects in the presence of non-zero images, having null projections along the considered directions. Such images are known as ghosts. The presence of ghosts was the input to investigate which parts of the object could be anyway reconstructed; this problem has been developed in [2-4].

In binary tomography, however, one can exploit the upper bound on the number of allowed grey levels in order to get some extra conditions. This has been done by Brunetti, Dulio and Peri in [5] (and later generalized to higher dimensions in [6]), who prove that just four suitably chosen valid directions guarantee uniqueness of reconstruction. Their main theorem is reported and explained in Section 3.

Starting from that result, we want to address another common issue which arises when facing the real world: that of stability, namely, what happens when a small perturbation affects four directions which ensure uniqueness. This problem is closely related to that of the presence of noise. In this paper, we prove that sets ensuring uniqueness are stable under the action of a small lattice perturbation.

The paper is organized as follows. In Section 2 we recall the main definitions concerning discrete tomography and lattice directions. In Section 3 the main uniqueness theorem of [5] is reported, while Section 4 shows the new results concerning stability. In Section 5 examples are depicted and commented; Section 6 focuses on possible further work and concludes the paper. 


\section{Definitions}

\subsection{Main models for discrete tomography}

We restrict our investigation to the two-dimensional case, so the object we aim to reconstruct is an image, occupying a subset of the lattice $\mathbb{Z}^{2}$, say a rectangular grid $\mathcal{A}$. We can therefore say that an image is a map $f: \mathcal{A} \rightarrow \mathbb{Z}$. By an abuse of notation, we will sometimes say that we reconstruct $\mathcal{A}$ instead of $f$.

The image is represented as a column vector $\mathbf{x} \in \mathbb{R}^{N}$, where $N$ is the number of the pixels composing the image. The projection data are collected in a vector $\mathbf{p} \in \mathbb{R}^{M}$, where $M$ corresponds to the sum of the lengths of the arrays gathering the measurements along each direction. So, the tomographic reconstruction problem is modeled as an equivalent linear system

$$
A \mathbf{x}=\mathbf{p},
$$

where $A$ is the $M \times N$ matrix such that its entry $a_{i j}$ is related to the contribution that the $j$-th pixel gives to the $i$-th equation. In general, such an approach is severely underdetermined, and many solutions are allowed for a given set of projections. Usually this leads to rely on iterative methods, such as ART, DART and SIRT (see for instance [7]).

Different ways of computing $a_{i j}$ s give different models for discrete tomography. We recall the two we will employ throughout the paper; in both of them, a pixel is considered to be a-dimensional, namely, collapsed into a point of the lattice, say its bottom-left corner.

In the discrete strip model (see Figure 1(a)), X-rays are strips of given width $w$. Here $a_{i j}=1$ if the pixel $x_{j}$ belongs to the $i$-th strip, and $a_{i j}=0$ otherwise. Therefore, $p_{i}$ sums the values of the lattice points falling in the corresponding strip.

For $w \rightarrow 0$, we get the grid model (see Figure 1(b)), where therefore X-rays are lattice lines. Consequently, $a_{i j}=1$ if the pixel $x_{j}$ belongs to the $i$-th line, and $a_{i j}=0$ otherwise. This model is the basic discrete theoretical model.

\subsection{Valid directions}

As previously said, the presence of ghosts causes ambiguities in the reconstruction. Ghosts' non-null pixels form the so-called (weakly) bad configurations, namely, pairs of sets of pixels having the same projections along the considered directions (see Figure 2 for an example). The presence of weakly bad configurations prevents an image from being uniquely reconstructed. We call $S$-bad configuration a bad configuration associated to a set $S$ of directions. If pixels can be counted with multiplicity greater than one, we deal with $S$-weakly bad configurations.

It is known from [8] that a grid $\mathcal{A}$ is uniquely determined by a set $S$ of directions if and only if no $S$-bad configuration can be constructed inside $\mathcal{A}$.

However, uniqueness can be trivially achieved, even with just one direction: for instance, if one takes a direction $u$ whose slope is such that each line parallel to $u$ has

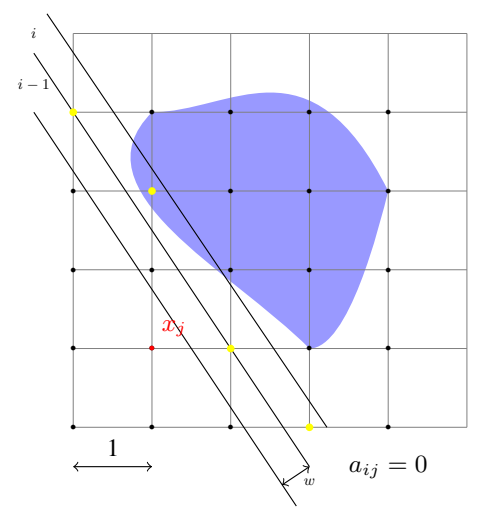

(a)

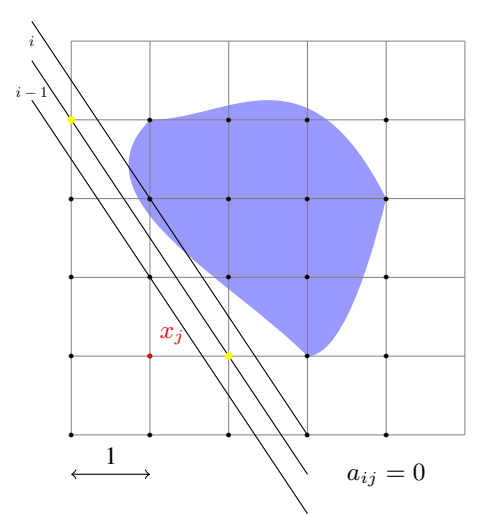

(b)

Figure 1. Different models for discrete tomography. (a) The discrete strip model. (b) The discrete line model, or grid model.

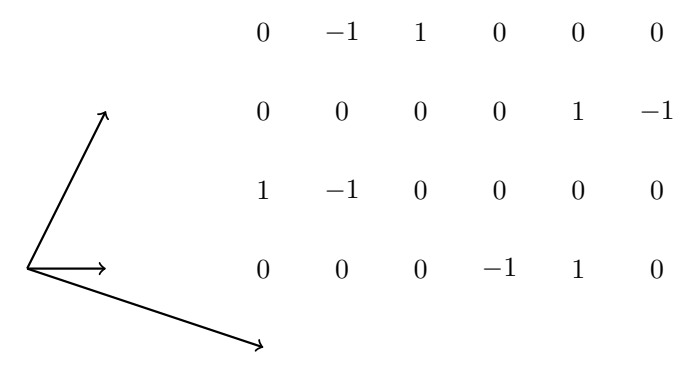

Figure 2. A ghost associated to the three depicted directions. The non-null pixels are the ones of the associated bad configuration.

at most one intersection with the grid $\mathcal{A}$, then the resulting linear system (1) has only one solution (see Figure 3). In real tomographic applications, however, non-valid directions are undesirable, since each detector should collect information point by point. We therefore consider a different approach.

In 2001, Hajdu and Tijdeman [9] introduced an algebraic approach to discrete tomography, which shows how ghosts can be treated in terms of polynomials. 


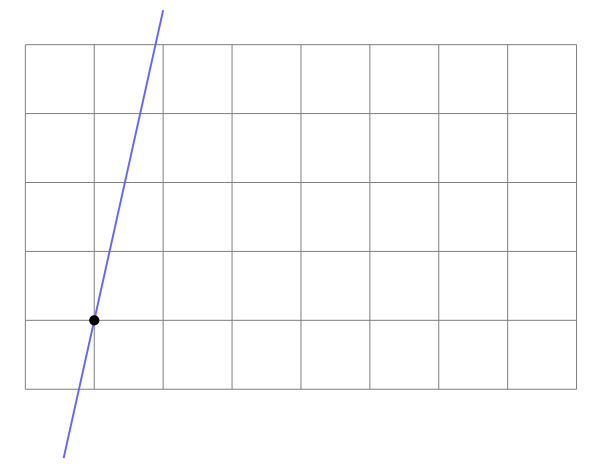

Figure 3. A single direction can ensure uniqueness of reconstruction.

Fix a lattice grid $\mathcal{A}=\left\{\left(z_{1}, z_{2}\right) \in \mathbb{Z}^{2} \mid 0 \leq z_{1}<m, 0 \leq\right.$ $\left.z_{2}<n\right\}$. A direction is modeled as a pair $(a, b)$ of coprime integers; coordinate directions are represented by the pairs $(1,0)$ (the horizontal one) and $(0,1)$ (the vertical one). We can assume without loss of generality that $a \geq 0$.

Definition 1 A set $S=\left\{\left(a_{r}, b_{r}\right) \mid r=1, \ldots, d\right\}$ of d lattice directions is said to be valid for the grid $\mathcal{A}$ if

$$
\sum_{r=1}^{d} a_{r}<m \quad \text { and } \quad \sum_{r=1}^{d}\left|b_{r}\right|<n .
$$

Therefore, valid sets are the ones that allow us to construct a ghost inside the grid.

\section{A uniqueness result for binary tomography}

Since valid sets of directions imply the presence of bad configurations inside the image, they cannot lead to uniqueness of reconstruction. To do so, we need to add other constraints. A commonly employed one is the prior knowledge of the number of grey levels of the image; in particular, one is often interested in detecting the presence or the absence of a body, reflecting in the presence of just two shades, say black and white. This is the framework of binary tomography.

This kind of prior knowledge reveals to be fundamental in order to get uniqueness results with valid directions. The result we are going to recall, due to Brunetti, Dulio and Peri ([5]), characterizes the smallest sets of binary uniqueness, namely, sets of valid directions which ensure uniqueness of reconstruction for binary images. Such sets are composed of just four directions, built in a peculiar way; we now define the tools which will be employed in the characterization theorem.

First of all, sets $S$ of directions have to satisfy a necessary condition: one of the directions has to be the sum of the other three, or the sum of two of them must equal the sum of the other two. This means that $S=\left\{u_{1}, u_{2}, u_{3}, u_{4}=\right.$ $\left.u_{1}+u_{2} \pm u_{3}\right\}$. Note that $u_{4}$ has to be a direction, namely, a pair of coprime integers, so not all triples $u_{1}, u_{2}, u_{3}$ are allowed. This condition on the directions reflects in the fact that a minimal bad configuration associated to the set $S$ has a pixel which is counted twice, so its value is 2 or -2 . This is not allowed in a binary fashion, so such a bad configuration cannot be constructed inside a binary grid.

Set $u_{r}=\left(a_{r}, b_{r}\right), r=1,2,3,4$, and

$$
\sum_{r=1}^{4} a_{r}=: h, \quad \sum_{r=1}^{4}\left|b_{r}\right|=: k .
$$

Consider the set $D= \pm S \cup \hat{S}$, where

$$
\pm S=\left\{ \pm u_{1}, \pm u_{2}, \pm u_{3}, \pm u_{4}\right\}
$$

and

$$
\hat{S}=\left\{ \pm\left(u_{1}-u_{4}\right), \pm\left(u_{2}-u_{4}\right), \pm\left(u_{1}+u_{2}\right)\right\} .
$$

Note that the set $D$ is a set of pairs, so its elements are not necessarily directions.

Define the subsets $A, B$ of $D$ as follows:

$$
\begin{aligned}
& A:=\{(a, b) \in D|| a|>| b \mid\}, \\
& B:=\{(a, b) \in D|| b|>| a \mid\} .
\end{aligned}
$$

Moreover, if $|a|=|b|$, for some $(a, b) \in D$, we then include $(a, b)$ in $A$ if $\min \{m-h, n-k\}=m-h$, while $(a, b) \in B$ otherwise. Thus $D$ is the disjoint union of $A$ and $B$, where one of the two sets may be empty.

We can now state the uniqueness theorem.

Theorem 2 ([5], Theorem 6) Let $S=\left\{u_{1}, u_{2}, u_{3}, u_{4}=\right.$ $\left.u_{1}+u_{2} \pm u_{3}\right\}$ be a valid set for the lattice grid $\mathcal{A}=\left\{\left(z_{1}, z_{2}\right) \in\right.$ $\left.\mathbb{Z}^{2} \mid 0 \leq z_{1}<m, 0 \leq z_{2}<n\right\}$. Then every binary image defined on $\mathcal{A}$ is uniquely determined if and only if

$$
\begin{gathered}
\min _{(a, b) \in A}|a| \geq \min \{m-h, n-k\}, \\
\min _{(a, b) \in B}|b| \geq \min \{m-h, n-k\},
\end{gathered}
$$

and

$$
\begin{array}{r}
m-h<n-k \Rightarrow \forall(a, b) \in B: \\
|a| \geq m-h \text { or }|b| \geq n-k, \\
n-k<m-h \Rightarrow \forall(a, b) \in A: \\
|a| \geq m-h \text { or }|b| \geq n-k,
\end{array}
$$

where, if one of the sets $A, B$ is empty, the corresponding condition (3) or (4) drops.

Denote by $\mathcal{S}(\mathcal{A})$ the collection of sets of binary uniqueness for a grid $\mathcal{A}$. We wish to exploit Theorem 2 to solve the linear system (1), which will have a unique binary solution.

\section{Stability results}

In view of applications, given a lattice grid $\mathcal{A}$, it would be desirable to have different sets from $\mathcal{S}(\mathcal{A})$, in order to select the set $S$ which better fits with the faced constraints. Therefore, we wish to investigate how $S$ could be changed without affecting uniqueness.

A first simple but useful result is the following. 
Theorem 3 Let $\mathcal{A}=\left\{\left(z_{1}, z_{2}\right) \in \mathbb{Z}^{2} \mid 0 \leq z_{1}<m, 0 \leq z_{2}<\right.$ $n\}$ be a lattice grid, and let $S=\left\{u_{1}, u_{2}, u_{3}, u_{4}\right\} \in \mathcal{S}(\mathcal{A})$. Denote by $\mathbf{p}_{S}$ the projection vector obtained by employing the directions of $S$. Then there exist continuous intervals $J_{i}, i=1,2,3,4$, such that $u_{i} \in J_{i}$, and, for all $S^{\prime}=\left\{u_{i}^{\prime} \mid\right.$ $\left.u_{i}^{\prime} \in J_{i}\right\}$, the linear system $A \mathbf{x}=\mathbf{p}_{S^{\prime}}$, has only one solution, which equals the unique binary solution of $A \mathbf{x}=\mathbf{p}_{S}$.

Proof. Slightly changing the directions in $S$, we can always obtain a set $S^{\prime}$ formed by directions which are not valid for the $m \times n$ lattice grid $\mathcal{A}$. This can be easily achieved by replacing some (possibly all) $u_{i}=\left(a_{i}, b_{i}\right) \in S$, $i=1,2,3,4$, with $u_{i}^{\prime}=\left(a_{i}^{\prime}, b_{i}^{\prime}\right)$ (see Figure 4), such that

$$
\frac{b_{i}^{\prime}}{a_{i}^{\prime}}=\tan \gamma=\frac{w}{L}= \begin{cases}\frac{b_{i}}{n\left(a_{i}^{2}+b_{i}^{2}\right)} & \text { if } b_{i}>a_{i} \\ \frac{a_{i}}{m\left(a_{i}^{2}+b_{i}^{2}\right)} & \text { if } b_{i}<a_{i} .\end{cases}
$$

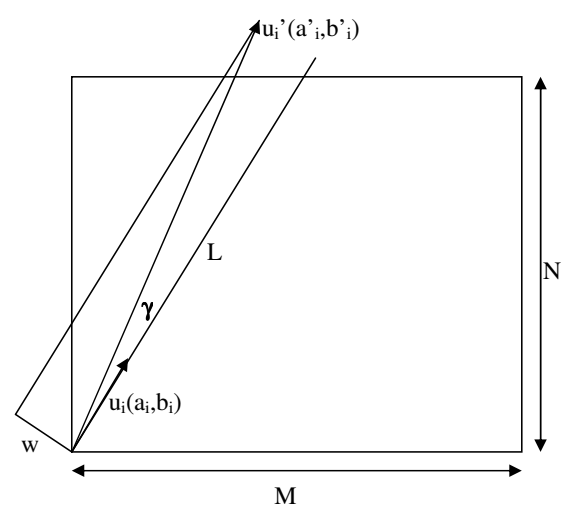

Figure 4. Angle $\gamma$ between a non valid direction $u^{\prime}=\left(a_{i}^{\prime}, b_{i}^{\prime}\right)$ obtained by a slight change of $u_{i}=\left(a_{i}, b_{i}\right) \in S$.

In fact, in this case, each line in direction $u_{i}^{\prime}$ intersects the grid in a segment completely included in the strip bounded by two adjacent lattice lines parallel to the direction $u_{i}$. Since $S^{\prime}$ contains non-valid directions, then $S^{\prime}$ does not satisfy condition (2). Therefore, $S^{\prime}$ is a set of general (not necessarily binary) uniqueness, meaning that the linear system $A \mathbf{x}=\mathbf{p}_{S^{\prime}}$ has just one solution.

Remark 4 We have already said that non-valid directions are not desirable. However, instead of regarding Theorem 3 as a stable noise-free reconstruction result, we could interpret it as an exact reconstruction result for $X$-ray affected by some noise.

As previously observed, in the grid model X-rays count the number of lattice points belonging to lines having rational slopes. In view of real applications we should also remark that, moving from source to detector, X-rays can be affected by deviations from the straight line paths, due to mechanical and physical phenomena. This means that a single X-ray, in fact, counts more points than in the usual grid model, since its trajectory meets different lattice lines. Trajectories could be highly different from ray to ray, so, in order to model the various behaviors, we assume that each $X$-ray path is confined in a strip of prescribed width $w$, and that each point in the strip cannot be visited twice. This means that the number of lattice points included in a given strip represents an upper bound on the corresponding detected projection.

This can be interpreted as a discrete strip model of the continuous approach, where a ray issuing from a given source $P$ could be detected by any point of the line segment $L_{P}$ of length $w$, and axis of symmetry passing through $P$. In term of back-projection, the strip orthogonal to $L_{P}$ is spanned, and consequently a noise, depending on the width of the strip, is introduced with respect to the corresponding grid-model reconstruction process. This suggests to interpret the discrete strip model as a kind of noisy grid model, where the width $w$ of the strips corresponds to the amount of noise that affects the grid model projections.

Now we want to show that a small discrete perturbation of a valid set of uniqueness, that preserves the validity of the employed directions, also preserves uniqueness. To this, we need to introduce a notion of lattice perturbation of a lattice direction.

Definition 5 Let $d=\left(d_{1}, d_{2}\right)$ be a given lattice direction. For each $\epsilon=\frac{a}{b} \in \mathbb{Q} \cap[0,1]$, and $\delta_{x}, \delta_{y} \in\{0,1\}$, the $\epsilon$ lattice perturbation of $d$ is the vector $d\left(\epsilon, \delta_{x}, \delta_{y}\right)$, parallel to $d+\delta_{x}(\epsilon, 0)+\delta_{y}(0, \epsilon)$, given by

$$
d\left(\epsilon, \delta_{x}, \delta_{y}\right)=\left(d_{1} b+a \delta_{x}, d_{2} b+a \delta_{y}\right) .
$$

Note that

$$
\frac{d_{2}}{d_{1}+\epsilon} \leq \frac{d_{2}}{d_{1}} \leq \frac{d_{2}+\epsilon}{d_{1}}
$$

and also

$$
\begin{aligned}
\frac{d_{2}}{d_{1}+\epsilon} & \leq \frac{d_{2}}{d_{1}+\epsilon \delta_{x}} \leq \frac{d_{2} b}{d_{1} b+a \delta_{x}} \\
& \leq \frac{d_{2} b+a \delta_{y}}{d_{1} b+a \delta_{x}} \leq \frac{d_{2} b+a \delta_{y}}{d_{1} b} \leq \frac{d_{2}+\epsilon}{d_{1}},
\end{aligned}
$$

so that the slope of $d\left(\epsilon, \delta_{x}, \delta_{y}\right)$ is sufficiently close to the slope of $d$. Therefore, $d\left(\epsilon, \delta_{x}, \delta_{y}\right)$ is a lattice vector (not necessarily a lattice direction), bounded in a small angular neighbor of $d$.

Theorem 6 Let $\mathcal{A}$ be a lattice grid, and let $S=$ $\left\{u_{1}, u_{2}, u_{3}, u_{4}\right\} \in \mathcal{S}(\mathcal{A})$. Let $S^{\prime}=\left\{u_{1}^{\prime}, u_{2}^{\prime}, u_{3}^{\prime}, u_{4}^{\prime}\right\}$ such that $u_{1}^{\prime}=u_{1}\left(\epsilon, \delta_{x}, \delta_{y}\right), u_{2}^{\prime}=u_{2}\left(\epsilon, \delta_{x}, \delta_{y}\right), u_{3}^{\prime}=u_{3}$ and $u_{4}^{\prime}=u_{1}^{\prime}+u_{2}^{\prime}+u_{3}^{\prime}$ if $u_{4}=u_{1}+u_{2}^{\prime}+u_{3}$, or $u_{4}^{\prime}=u_{1}^{\prime}+u_{2}^{\prime}-u_{3}^{\prime}$ if $u_{4}=u_{1}+u_{2}-u_{3}$. If $S^{\prime}$ is a set of valid directions for $\mathcal{A}$, then $S^{\prime} \in \mathcal{S}(\mathcal{A})$.

Proof. Assume $u_{r}=\left(a_{r}, b_{r}\right), u_{r}^{\prime}=\left(a_{r}^{\prime}, b_{r}^{\prime}\right)$, and consider the case when $u_{4}=u_{1}+u_{2}+u_{3}$. We have

$$
\begin{aligned}
& h^{\prime}=2\left(a_{1}^{\prime}+a_{2}^{\prime}+a_{3}^{\prime}\right) \geq 2\left(a_{1}+a_{2}+a_{3}\right)=h \\
& k^{\prime}=2\left(\left|b_{1}^{\prime}\right|+\left|b_{2}^{\prime}\right|+\left|b_{3}^{\prime}\right|\right) \geq 2\left(\left|b_{1}\right|+\left|b_{2}\right|+\left|b_{3}\right|\right)=k,
\end{aligned}
$$

and consequently

$$
\begin{aligned}
\min \left\{m-h^{\prime}, n-k^{\prime}\right\} & \leq \min \{m-h, n-k\}, \\
\max \left\{m-h^{\prime}, n-k^{\prime}\right\} & \leq \max \{m-h, n-k\} .
\end{aligned}
$$


Now, consider the set $D^{\prime}= \pm S^{\prime} \pm \widehat{S^{\prime}}=A^{\prime} \cup B^{\prime}$. For each $d^{\prime}=\left(d_{1}^{\prime}, d_{2}^{\prime}\right) \in D^{\prime}$ let $d=\left(d_{1}, d_{2}\right) \in D$ be the corresponding vector. Note that $d_{1}^{\prime} \geq d_{1}$ and $d_{2}^{\prime} \geq d_{2}$.

Now, let $d^{\prime} \in D^{\prime}=A^{\prime} \cup B^{\prime}$. There are several cases to be considered.

Case 1: $d^{\prime} \in A^{\prime}$. Since $S \in \mathcal{S}(\mathcal{A})$, we have two subcases.

Case 1a: If $d \in A$, then $\left|d_{1}\right| \geq \min \{m-h, n-k\}$, so that

$\left|d_{1}^{\prime}\right| \geq\left|d_{1}\right| \geq \min \{m-h, n-k\} \geq \min \left\{m-h^{\prime}, n-k^{\prime}\right\}$.

Case $1 b$ : If $d \in B$, then $\left|d_{2}\right| \geq \min \{m-h, n-k\}$, so that

$\left|d_{2}^{\prime}\right| \geq\left|d_{2}\right| \geq \min \{m-h, n-k\} \geq \min \left\{m-h^{\prime}, n-k^{\prime}\right\}$

and, since $d^{\prime} \in A^{\prime},\left|d_{1}^{\prime}\right| \geq\left|d_{2}^{\prime}\right|$, so that $\left|d_{1}^{\prime}\right| \geq \min \left\{m-h^{\prime}, n-\right.$ $\left.k^{\prime}\right\}$ as in subcase $1 a$.

Therefore, for all $d^{\prime} \in A^{\prime}$, it is

$$
\left|d_{1}^{\prime}\right| \geq \min \left\{m-h^{\prime}, n-k^{\prime}\right\},
$$

and consequently

$$
\min _{\left(a^{\prime}, b^{\prime}\right) \in A^{\prime}}\left|a^{\prime}\right| \geq \min \left\{m-h^{\prime}, n-k^{\prime}\right\},
$$

so that condition (3) in Theorem 2 holds.

Case 2: $d^{\prime} \in B^{\prime}$. Since $S \in \mathcal{S}(\mathcal{A})$, there are two subcases again.

Case $2 a$ : If $d \in A$, then $\left|d_{1}\right| \geq \min \{m-h, n-k\}$, so that

$\left|d_{1}^{\prime}\right| \geq\left|d_{1}\right| \geq \min \{m-h, n-k\} \geq \min \left\{m-h^{\prime}, n-k^{\prime}\right\}$

and, since $d^{\prime} \in B^{\prime},\left|d_{2}^{\prime}\right| \geq\left|d_{1}^{\prime}\right|$, so that $\left|d_{2}^{\prime}\right| \geq \min \left\{m-h^{\prime}, n-\right.$ $k^{\prime}$.

Case 2b: If $d \in B$, then $\left|d_{2}\right| \geq \min \{m-h, n-k\}$, so that $\left|d_{2}^{\prime}\right| \geq\left|d_{2}\right| \geq \min \{m-h, n-k\} \geq \min \left\{m-h^{\prime}, n-k^{\prime}\right\}$,

as in subcase $2 a$.

Therefore, for all $d^{\prime} \in B^{\prime}$, it is

$$
\left|d_{2}^{\prime}\right| \geq \min \left\{m-h^{\prime}, n-k^{\prime}\right\}
$$

and consequently

$$
\min _{\left(a^{\prime}, b^{\prime}\right) \in B^{\prime}}\left|b^{\prime}\right| \geq \min \left\{m-h^{\prime}, n-k^{\prime}\right\},
$$

so that condition (4) in Theorem 2 holds.

Now, suppose that $\min \left\{m-h^{\prime}, n-k^{\prime}\right\}=m-h^{\prime}$, and consider any $d^{\prime} \in B^{\prime}$. If $d \in A$, then, by condition (3) in Theorem 2, we have $\left|d_{1}\right| \geq \min \{m-h, n-k\}$, so that

$$
\begin{aligned}
\left|d_{1}^{\prime}\right| \geq\left|d_{1}\right| & \geq \min \{m-h, n-k\} \\
& \geq \min \left\{m-h^{\prime}, n-k^{\prime}\right\}=m-h^{\prime} .
\end{aligned}
$$

If $d \in B$, and $\min \{m-h, n-k\}=m-h$, then, by condition (5) in Theorem 2, it may be $\left|d_{1}\right| \geq m-h$, so that

$$
\left|d_{1}^{\prime}\right| \geq\left|d_{1}\right| \geq m-h \geq m-h^{\prime},
$$

or $\left|d_{2}\right| \geq n-k$, so that

$$
\left|d_{2}^{\prime}\right| \geq\left|d_{2}\right| \geq n-k \geq n-k^{\prime} .
$$

If $d \in B$, and $\min \{m-h, n-k\}=n-k$, then, by condition (4) in Theorem 2, we have $\left|d_{2}\right| \geq n-k$, so that

$$
\left|d_{2}^{\prime}\right| \geq\left|d_{2}\right| \geq n-k \geq n-k^{\prime} .
$$

Therefore, we have

$$
\forall d^{\prime} \in B^{\prime}:\left|d_{1}^{\prime}\right| \geq m-h^{\prime} \text { or }\left|d_{2}^{\prime}\right| \geq n-k^{\prime}
$$

and condition (5) in Theorem 2 holds.

Similarly, if $\min \left\{m-h^{\prime}, n-k^{\prime}\right\}=n-k^{\prime}$, consider any $d^{\prime} \in A^{\prime}$. If $d \in A$ and $\min \{m-h, n-k\}=m-h$, then, by condition (3) in Theorem 2, we have $\left|d_{1}\right| \geq m-h$, so that

$$
\left|d_{1}^{\prime}\right| \geq\left|d_{1}\right| \geq m-h \geq m-h^{\prime} .
$$

If $d \in A$ and $\min \{m-h, n-k\}=n-k$, then, by condition (6) in Theorem 2 , it could be $\left|d_{1}\right| \geq m-h$, so that

$$
\left|d_{1}^{\prime}\right| \geq\left|d_{1}\right| \geq m-h \geq m-h^{\prime},
$$

or $\left|d_{2}\right| \geq n-k$, so that

$$
\left|d_{2}^{\prime}\right| \geq\left|d_{2}\right| \geq n-k \geq n-k^{\prime} .
$$

If $d \in B$, then, by condition (4) in Theorem 2, we have $\left|d_{2}\right| \geq \min \{m-h, n-k\}$, so that

$$
\begin{aligned}
\left|d_{2}^{\prime}\right| \geq\left|d_{2}\right| & \geq \min \{m-h, n-k\} \\
& \geq \min \left\{m-h^{\prime}, n-k^{\prime}\right\}=n-k^{\prime} .
\end{aligned}
$$

Therefore, we have

$$
\forall d^{\prime} \in A^{\prime}:\left|d_{1}^{\prime}\right| \geq m-h^{\prime} \text { or }\left|d_{2}^{\prime}\right| \geq n-k^{\prime},
$$

and condition (6) in Theorem 2 holds.

A similar proof works for $u_{4}=u_{1}+u_{2}^{\prime}-u_{3}$.

Theorem 7 Let $S=\left\{u_{i}=\left(a_{i}, b_{i}\right) \mid i=1,2,3,4\right\}$ be a set of lattice directions, and $r_{1}, r_{2}$ be such that

$$
\begin{array}{ll}
a_{1}=\min _{i} a_{i} & b_{1}=\min _{i} b_{i} \\
a_{2}=a_{1}+r_{1}, & b_{2}=b_{1}+s_{1}, \\
a_{3}=a_{1}+r_{2}, & b_{3}=b_{1}+s_{2}, \\
a_{4}=a_{1}+a_{2}+a_{3} & b_{4}=b_{1}+b_{2}+b_{3} \\
r_{1}+r_{2} \geq \frac{m-7 a_{1}}{2} & s_{1}+s_{2} \geq \frac{n-7 b_{1}}{2} .
\end{array}
$$

Then $S \in \mathcal{S}(\mathcal{A})$.

Proof. The set $S$ is of the form $S=\left\{u_{1}, u_{2}, u_{3}, u_{4}=u_{1}+\right.$ $\left.u_{2} \pm u_{3}\right\}$, therefore we must prove that the assumptions of Theorem 2 hold.

Consider the set $\widehat{S}=\left\{ \pm\left(u_{1}-u_{4}\right), \pm\left(u_{2}-u_{4}\right), \pm\left(u_{1}+u_{2}\right)\right\}$. Since $a_{1}=\min _{i} a_{i}$, and $b_{1}=\min _{i} b_{i}$, then, for each $\widehat{s}=$ $\left(\widehat{s}_{1}, \widehat{s}_{2}\right) \in \widehat{S}$, we have $\widehat{s}_{1} \geq a_{1}$ and $\widehat{s}_{2} \geq b_{1}$. Therefore, for any vector $d=\left(d_{1}, d_{2}\right) \in D= \pm S \cup \widehat{S}=A \cup B$, it results $d_{1} \geq a_{1}$ and $d_{2} \geq b_{1}$.

Now, note that

$$
h=a_{1}+a_{2}+a_{3}+a_{4}=6 a_{1}+r_{1}+r_{2}
$$

and

$$
k=b_{1}+b_{2}+b_{3}+b_{4}=6 b_{1}+s_{1}+s_{2} \text {. }
$$


Therefore, it results

$$
\begin{aligned}
m-h & =m-6 a_{1}-r_{1}-r_{2} \leq a_{1} \\
n-k & =n-6 b_{1}-s_{1}-s_{2} \leq b_{1} .
\end{aligned}
$$

Assume that $\min \{m-h, n-k\}=m-h$. If $u_{1} \in A$, then

$$
a_{1} \geq b_{1} \geq n-k \geq m-h \text {. }
$$

Consequently, if $\left(d_{1}, d_{2}\right) \in A$, then $d_{1} \geq a_{1} \geq m-h$, and Condition (3) in Theorem 2 holds. If $\left(d_{1}, d_{2}\right) \in B$, then $d_{2} \geq b_{1} \geq m-h$, and Condition (4) in Theorem 2 holds. Moreover, $d_{1} \geq a_{1} \geq m-h$, so that the first inequality of Condition (6) in Theorem 2 holds.

Suppose now that $\min \{m-h, n-k\}=n-k$. If $u_{1} \in A$, then

$$
a_{1} \geq b_{1} \geq n-k \geq m-h .
$$

Consequently, if $\left(d_{1}, d_{2}\right) \in A$, then $d_{1} \geq a_{1} \geq n-k$, and Condition (3) in Theorem 2 holds. Moreover, $d_{2} \geq b_{1} \geq$ $n-k$, so that the second inequality of Condition (5) in Theorem 2 holds. If $\left(d_{1}, d_{2}\right) \in B$, then $d_{2} \geq b_{1} \geq n-k$, and Condition (4) in Theorem 2 holds. Moreover, $d_{1} \geq$ $a_{1} \geq m-h$, so that the first inequality of Condition (6) in Theorem 2 holds.

Therefore, all conditions in Theorem 2 hold, and consequently $S \in \mathcal{S}(\mathcal{A})$. $\square$

In the experimental section we will provide a few examples of lattice perturbations.

\section{Experimental results}

In the following experiments, performed in a noise-free context, we employed $\epsilon$ equal to 1 . This choice is due to the fact we reconstruct small images $(11 \times 11$ and $15 \times 15$, respectively), so that greater values for $a, b$ (recall that $\epsilon=$ $\frac{a}{b}$ ) make the set $S^{\prime}$ non-valid for the grid $\mathcal{A}$.

In the first experiment, we consider the set

$$
\begin{aligned}
S & =\left\{u_{1}, u_{2}, u_{3}, u_{1}+u_{2}-u_{3}\right\} \\
& =\{(1,5),(2,-3),(0,1),(3,1)\},
\end{aligned}
$$

which is a set of binary uniqueness for an $11 \times 11$ grid. For $\epsilon=1$ and a suitable choice of the values of $\delta_{x} \mathrm{~s}$ and $\delta_{y} \mathrm{~s}$, the first two directions are modified as follows:

$$
\begin{aligned}
u_{1}=(1,5) \mapsto u_{1}^{\prime} & =(1 \cdot 1+1 \cdot 1,5 \cdot 1+1 \cdot 0) \\
& =(2,5), \\
u_{2}=(2,-3) \mapsto u_{2}^{\prime} & =(2 \cdot 1+1 \cdot 1,-3 \cdot 1+1 \cdot 1) \\
= & (3,-2) .
\end{aligned}
$$

The third direction of the new set $S^{\prime}$ is the same as the third one of set $S$, and so we get

$$
u_{4}^{\prime}=u_{1}^{\prime}+u_{2}^{\prime}-u_{3}^{\prime}=(5,2) .
$$

The set $S^{\prime}=\{(2,5),(3,-2),(0,1),(5,2)\}$ is again a set of binary uniqueness for $\mathcal{A}$. An example of reconstruction is shown in Figure 5.

In the second experiment, we deal with an $15 \times 15$ grid $\mathcal{A}$, and initial set of uniqueness is

$$
\begin{aligned}
S & =\left\{u_{1}, u_{2}, u_{3}, u_{1}+u_{2}-u_{3}\right\} \\
& =\{(1,-1),(3,-5),(0,1),(4,-7)\} .
\end{aligned}
$$

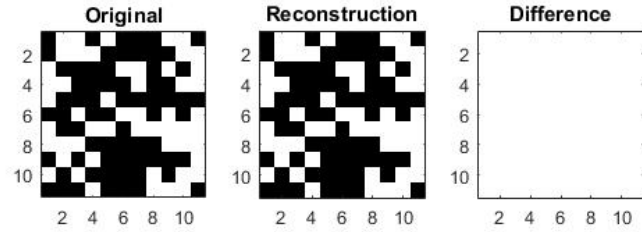

(a)
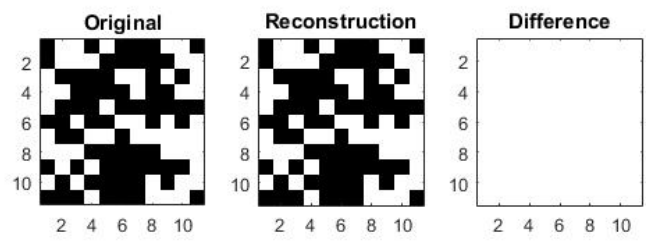

(b)

Figure 5. Reconstruction performed with different sets of directions on an $11 \times 11$ grid. In each line, the leftmost image is the original phantom, the central image is the reconstructed one, and the rightmost image shows the difference between the previous two (white pixel means exactly reconstructed pixel). (a) $S=\{(1,5),(2,-3),(0,1),(3,1)\}$. (b) $S^{\prime}=$ $\{(2,5),(3,-2),(0,1),(5,2)\}$.

Also in this case, we consider $\epsilon=1$; the second direction is not modified (namely, we choose $\delta_{x}=\delta_{y}=0$ in that case), while the first one becomes

$$
u_{1}=(1,-1) \mapsto u_{1}^{\prime}=(2,-1) .
$$

The new set is therefore

$$
S^{\prime}=\{(2,-1),(3,-5),(0,1),(5,-7)\} .
$$

Again, it satisfies all conditions of Theorem 2, so that $S^{\prime}$ is a set of binary uniqueness for $\mathcal{A}$. Figure 6 shows an example.

\section{Conclusions and new directions of research}

In this paper we have proven some results concerning the stability of sets of uniqueness in binary tomography, together with some experimental results. Sets of four directions, ensuring uniqueness for binary images defined on a 


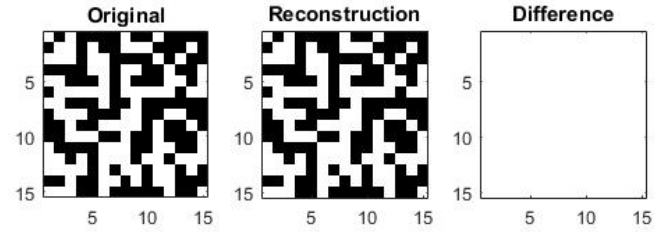

(a)

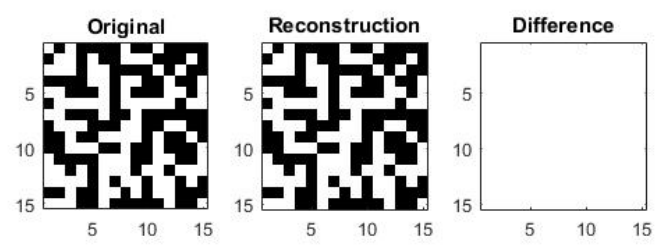

(b)

Figure 6. Reconstruction performed with different sets of directions on a $15 \times 15$ grid. (a) $S=\{(1,-1),(3,-5),(0,1),(4,-7)\}$. (b) $S^{\prime}=\{(2,-1),(3,-5),(0,1),(5,-7)\}$.

given lattice grid $\mathcal{A}$, are shown to be robust with respect to lattice perturbations. This could represent an interesting way of recovering sets of binary uniqueness, whose complete enumeration is still missing (see for instance [10]).

Further work can be done. First of all, noise could be introduced in the reconstruction process, so one has to face modifications not only of the lattice directions, but also in the entries of the projection vector $\mathbf{p}$.

Also, it would be interesting to combine the obtained stability results with another kind of investigation, relying on some preliminary results provided in [11, Theorem 2.5 and Corollary 2.1], which in turn rely on [12]. Here, the binary rounding of the solution obtained by pseudoinversion (via iterative methods) is taken into account, and different sets of binary uniqueness may play a role in the speed of convergence of the iterations.

\section{References}

[1] J. Radon, Ber. Verh. Sächs. Akad. Wiss. Leipzig Math.-Phys. Kl. pp. 262-277 (1917)

[2] P. Dulio, A. Frosini, S.M. Pagani, in Discrete geometry for computer imagery (Springer, Cham, 2014), Vol. 8668 of Lecture Notes in Comput. Sci., pp. 285296

[3] P. Dulio, A. Frosini, S.M. Pagani, Inverse Problems 31, 125011 (2015)

[4] P. Dulio, A. Frosini, S.M. Pagani, LNCS pp. 105116 (2016)

[5] S. Brunetti, P. Dulio, C. Peri, Discrete Appl. Math. 161, 2281 (2013)

[6] S. Brunetti, P. Dulio, C. Peri, Discrete Appl. Math. 183, 20 (2015)

[7] K.J. Batenburg, J. Sijbers, IEEE Trans. Image Process. 20, 2542 (2011)

[8] P.C. Fishburn, J.C. Lagarias, J.A. Reeds, L.A. Shepp, Discrete Math. 91, 149 (1991)

[9] L. Hajdu, R. Tijdeman, J. Reine Angew. Math. 534, 119 (2001)

[10] S. Brocchi, P. Dulio, S.M. Pagani, Recent Advances in Systems, in: Recent Advances in Electrical Engineering Series 52, 393 (2015)

[11] S.M. Pagani, From uniqueness results to reconstruction and characterization algorithms in discrete tomography (PhD thesis, Politecnico di Milano, 2016)

[12] K.J. Batenburg, W. Fortes, L. Hajdu, R. Tijdeman, Discrete Appl. Math. 161, 2236 (2013) 